\title{
Dietas locales, programas globales ¿favorecen la nutrición de las mujeres, niños y niñas? El caso de la comunidad de San Clemente, provincia de Imbabura
}

\author{
Laura Boada Molina*
}

\begin{abstract}
RESUMEN

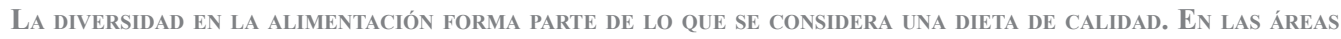
RURALES LAS FAMILIAS TIENEN ACCESO A ESTE TIPO DE DIETAS GRACIAS A LOS ALIMENTOS DE PRODUCCIÓN LOCAL PARA el autoconsumo. Sin embargo, no Siempre esta diversidad Se refleja en una dieta diversa. Por otra Parte, CUANDO LA MALNUTRICIÓN O DESNUTRICIÓN CRÓNICA ES EVIDENTE EN NIÑOS MENORES DE CINCO AÑOS, LOS PROGRAMAS ESTATALES INTENTAN PALIAR ESTE PROBLEMA A TRAVÉS DE SUPLEMENTOS QUE NO EN TODOS LOS CASOS LOGRAN SER UN COMPONENTE DEFINITORIO DE UNA MEJOR ALIMENTACIÓN Y POR ENDE DE UNA MEJOR NUTRICIÓN. EL PRESENTE TRABAJO BUSCA APORTAR UNA PERSPECTIVA CRÍTICA DE ESTAS DOS PREMISAS TOMANDO COMO CASO DE ESTUDIO LA COMUNIDAD de San Clemente, localizada en la sierra norte del Ecuador, provincia de Imbabura.
\end{abstract}

Palabras Claves: alimentación - nutrición RURal - diversidad alimentaria - micronutrientes.

\section{Abstract}

DIVERSITY IN FOOD IS CONSIDERED PART OF WHAT CONSTITUTES A QUALITY DIET. IN RURAL AREAS, FAMILIES HAVE ACCESS TO THIS TYPE OF DIET THANKS TO LOCAL PRODUCTION OF FOODS.. HOWEVER, THE PRESENCE OF CROP DIVERSITY IS NOT ALWAYS REFLECTED IN A DIVERSE DIET. AdDITIONALly, WHEN MALNUTRITION OR CHRONIC MALNUTRITION IS EVIDENT IN CHILDREN UNDER FIVE YEARS OLD, STATE PROGRAMS AIM TO ALLEVIATE THIS PROBLEM THROUGH SUPPLEMENTS THAT DO NOT ALWAYS REPRESENT COMPONENTS OF A BETTER DIET NOR OF BETTER NUTRITION. THE PRESENT WORK ATTEMPTS

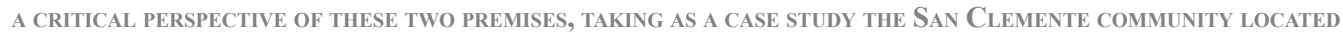
IN THE NORTHERN HIGHLANDS OF ECUADOR, IN THE PROVINCE OF IMBABURA.

KEYWORDS: FOOD - RURAL NUTRITION - FOOD DIVERSITY - MICRONUTRIENTS.

* Magister en Desarrollo Territorial Rural, Facultad Latinoamericana de Ciencias Sociales, FLACSO. Investigadora. Correo electrónico: laura.boada.molina@gmail.com 


\section{Introducción}

7 presente artículo tiene el objetivo de mostrar las elecciones de alimentos en tres madres y tres niños y niñas menores de cinco años, a través de la composición de su dieta. Así como también pretende abordar la ingesta de micronutrientes y la aceptabilidad de los mismos en individuos sujetos a programas paliativos para la malnutrición.

El estudio se llevó a cabo en la comunidad de San Clemente, perteneciente a la parroquia de Ambuquí, misma que presenta una alta diversidad de cultivos, por lo que se pensaría que esta diversidad es una precondición para que se refleje lo propio en la alimentación. A pesar de ello, tal y como manifiesta Messer (1995: 28-34) aunque los individuos se encuentren dentro de entornos ecológicamente similares -de alta diversidad de cultivos- unos sufren de desnutrición o malnutrición mientras que otros no.

Dicho lo anterior, pese a la alta diversidad de cultivos la parroquia de Ambuquí presenta un $29,03 \%$ de anémicos en edades de 6 meses a 59 meses de edad (anemia severa, moderada y leve). Para tratar este problema se realiza la suplementación a través del programa CHIS PAZ para mejorar la nutrición en niños y niñas menores de cinco años ( 0 a 59 meses de edad). Sin embargo las normas y prescripciones que conllevan estos métodos de suplementación no logran ser adoptadas por los hogares de manera adecuada. Pese a la diversidad de cultivos no existe un enfoque preventivo en la alimentación, a través del uso de alimentos de la localidad, por el contrario se da lo que se conoce como el proceso de medicalización de la alimentación.

\section{Propuesta teórica}

El ser humano en ocasiones podría preferir alimentos con mayor valor simbólico, aceptables organolépticamente. A pesar de ello, estos alimentos no siempre son nutricionalmente adecuados. Las poblaciones generalmente adoptan hábitos alimentarios de acuerdo a las elecciones de los individuos y grupos familiares y posteriormente las elecciones se adaptan al grupo comunitario o la sociedad. Sin embargo, aunque las poblaciones se encuentren geográficamente cercanas no siempre sus elecciones alimentarias son las mismas (Garine, 2002: 131).

Debemos señalar que la alimentación en toda población siempre es dinámica, sobre todo si ésta se encuentra ya vinculada fuertemente al mercado, según Lévi Strauss (1968), las modificaciones en los sistemas de autoconsumo describen gran parte de su estructura, organización e historia.(Ibíd.: 137).

Por otro lado, para abordar el tema de la introducción de "medicinas" como mecanismos para mejorar la nutrición en las áreas rurales, cabe citar el concepto de "medicalización de la alimentación" (Poulain, 2008), es decir qué términos, prácticas, reglas etc., reemplazan a las normas socialmente determinadas del acto alimentario por razones nutricionales basadas en conocimientos científicos. Podemos describir estos fenómenos por modificación alimentaria, utilizando el ejemplo de la sociedad urbana, interpretando a Garine (2002: 142) quien dice que estas sociedades urbanas debido al trabajo asalariado presentan una modificación en las prácticas alimentarias, y a través de ello modifican los alimentos que componen sus prácticas. Asimismo, en las áreas rurales de los países en vías de desarrollo las prácticas alimentarias se ven fuertemente influenciadas por el trabajo asalariado y la comercialización de cultivos de ciclo corto, modificación que se entendería como el proceso de externalización de la alimentación (Boada, 2014: 57-69).

Según Garine (2002: 165) el aumento en los ingresos da como resultado un mayor consumo de alimentos calóricos que provienen principalmente de las grasas. Tal es el caso de las áreas rurales en el Ecuador que adquieren nuevas prácticas de alimentación y consumo, y reemplazan alimentos

1 Este suplemento es distribuido a través de los Centros Infantiles del Buen Vivir (CIBV) y Creciendo con Nuestro Hijos (CNH) a escala nacional, beneficiando a alrededor de 400.000 niños y niñas. En la parroquia de Ambuquí el $24,72 \%$ de los niños fueron prescritos con este suplemento en el primer semestre del 2014 (Prevalencia de niños/as de 6 a 59 meses de edad, suplementados con micronutrientes en polvo, 2014). 
de mayor valor nutricional, por alimentos con mayor carga calórica provenientes de carbohidratos pobres en vitaminas como son las harinas refinadas, fideos y arroz blanco, por citar unos ejemplos.

A pesar de la gran diversidad en ciertas áreas donde prevalece la malnutrición y desnutrición, según Garine (2002: 166) las propuestas para mejorar el estado nutricional de la población pueden ir en detrimento de las prácticas culturales. Pese a ello, este es un precio que aparentemente se debe pagar, para acceder al mejoramiento de la calidad de vida en las áreas más vulnerables.

Por su parte Ellen Messer utiliza la tipología alimentaria, para hablar sobre los adecuados o inadecuados niveles de alimentación y sobre los patrones en la alimentación por los cuales persiste el problema del hambre. Esta tipología consta de tres tipos de dietas: 1) dieta básica, casi vegetariana; 2) dieta mejorada, el $15 \%$ de la energía proviene de alimentos de origen animal y 3) dieta completa y sana, en donde el $25 \%$ de la energía proviene de alimentos de origen animal (Messer, 2006: 32).

\section{El contexto}

En la encuesta de condiciones de vida del Ecuador (2005-2006) se expone que un $8.7 \%$ de los hogares ecuatorianos no accede a la canasta alimentaria básica, es decir a aquella que cubre los requerimientos calóricos diarios de aproximadamente 2000 calorías. Las familias adoptan estrategias tales como préstamos de alimentos en las tiendas. Sin embargo estas estrategias podrían ser insostenibles a largo plazo y dificultar el acceso a alimentación futura de ese hogar (ECV 2005 - 2006), en Calero, 2011: 28.

En el 2006 el 76\% de las familias en situación de inseguridad alimentaria se encontraban en las áreas rurales, y un poco más de la mitad se encontraba en la sierra rural (Ibíd.:33).

La desnutrición crónica según la UNICEF se trata del retardo de altura para la edad (A/E). Asociada normalmente a situaciones de pobreza, con consecuencias para el aprendizaje y menor desempeño económico. A nivel nacional el $23.9 \%$ de los niños y niñas menores de cinco años presenta desnutrición crónica. Mientras que en el área rural este porcentaje es de 31.9\% (ECV, 2014).

La nutrición es crucial en niños menores de cinco años, y es uno de los factores para el buen desempeño de los seres humanos en su edad adulta. No obstante, para lograrla es importante la disponibilidad de alimentos y que estos sean accesibles a las familias. A esta disponibilidad de alimentos se la entiende como la cantidad y la calidad indispensables para cubrir la necesidad alimenticia y nutricional de las familias, también conocida como seguridad alimentaria (INEC, 2008:5).

El Ministerio de Salud Pública revela que el IMC (Índice de Masa Corporal) es un indicador que se utiliza para mostrar el estado nutricional de una persona de entre 18 a 59 años. Si el resultado IMC es menor a 18.5 la persona posee bajo peso; si la persona arroja un resultado de entre 18.5 y 25 , la persona tiene un peso normal; mientras que si el índice es mayor o igual a 25 la persona se encuentra en el grupo de sobrepeso y obesidad. Los resultados según la ECV arrojaron que para el 2014 el $1.1 \%$ de la población a nivel de país tenía bajo peso (IMC $<18.5$ ). Mientras que el $34.9 \%$ de la población a nivel del país se encontraba en peso normal (IMC $>18.5<25$ ). Y finalmente el dato más relevante es el de la población con sobrepeso y obesidad, la misma que a nivel del país es del 64\% (IMC $\geq 25$ ). Para el caso del área rural este porcentaje es del 59.9\%. Mientras que para el área urbana es del $65.7 \%$.

De acuerdo a la encuesta ENIGHUR 2011-2012 el gasto corriente mensual de los hogares en alimentos y bebidas no alcohólicas es de \$584.496.341 es decir, del total del gasto corriente, este rubro representa el $24.4 \%$. Para el caso de las áreas rurales representa el $32 \%$ del total del gasto corriente mensual, mientras que para las áreas urbanas este representa el $22.6 \%$ del total del gasto corriente mensual. Es paradójico que en las áreas rurales, en dónde se produce la mayor proporción de alimentos, se destine un mayor porcentaje del gasto corriente mensual a este rubro. Por otro lado, es importante resaltar que los hogares más vulnerables, aquellos presentes en el decil 1 destinan un mayor porcentaje de su gasto corriente hacia el consumo de alimentos y bebidas no alcohólicas, el 42.3\%, mientras que el decil 10, el más rico, destina únicamente un $11.3 \%$. 
El pan y los cereales es el grupo de alimentos al cual se destina el mayor porcentaje del gasto corriente mensual en las áreas rurales (6.8\%); seguido del grupo de carnes frescas y congeladas (5.7\%).

\section{San Clemente}

La comunidad de San Clemente se encuentra localizada en la parroquia de Ambuquí, cantón Ibarra en la Provincia de Imbabura. Además de San Clemente, la parroquia de Ambuquí cuenta con las siguientes comunidades: Juncal, Carpuela, Chota, Rancho Chico, Chaupi Guaranguí, Rumipamba, Apangora, Las Mercedes y Lavanderos.

La parroquia de Ambuquí tiene como principal actividad económica a la agricultura (53\%). Según el Plan Desarrollo y Ordenamiento Territorial de la Parroquia Rural Ambuquí - Chota 2012 - 2025 San Clemente cuenta con alrededor de 19 especies cultivadas, entre aquellas de ciclo corto, semipermanente y permanente, entre las que se encuentran: fréjol, tomate riñón, pimiento, ají, maíz, zanahoria blanca, arveja, lechuga, col, caña de azúcar, sandía, limón, mandarina, naranja, granadilla, melón y babaco (Gobierno Autónomo descentralizado parroquial de Ambuquí-Chota, 2012: 140). Además de estos productos, los que se observaron durante la visita fueron: fréjol guandul, fréjol cabascango, camote, sambo, cebolla, zanahoria amarilla, lechuga y plátano.

De acuerdo con AGRECO al calcular el consumo de nutrientes en 406 familias de la parroquia de Ambuquí, del total de la población, solamente el 23\% consume el nivel recomendado de energía, el $17 \%$ de la población consume los requerimientos adecuados de proteínas, y la mayoría, el $30 \%$ de la población tiene una ingesta adecuada de carbohidratos (AGRECO, 2010).

\section{Propuesta metodológica}

Los estudios antropológicos sobre los problemas nutricionales a nivel de individuos en ocasiones resultan ser apropiados como complemento de aquellos que se ven reflejados en datos estadísticos, los cuáles muestran una generalidad que no necesariamente muestra lo que ocurre en todos los hogares. Se consideran como herramientas y métodos complementarios. Pero en este artículo para entender las prácticas locales en relación a métodos globales, se realizó en un nivel microsocial, tomando como unidad de análisis a los individuos y sus dietas.

Según Garine (2002: 161) normalmente algunas encuestas y otros métodos para identificar el consumo de alimentos, no consideran los alimentos que se consumen fuera del hogar, y únicamente toman en cuenta los alimentos que se preparan en el hogar. Para tener un acercamiento a las dietas de las familias dentro de la comunidad de San Clemente se utilizó el recordatorio de 24 horas. Este método consiste en preguntar a la persona encargada de preparar los alimentos en el hogar sobre los alimentos y bebidas que se consumieron las 24 horas previas a la entrevista, durante todas las comidas: previo al desayuno, en el desayuno, posterior al desayuno, en el almuerzo, posterior al almuerzo, en la cena y posterior a la cena.

Cabe aclarar que no se consideraron ni las medidas, ni los pesos de los alimentos consumidos; únicamente se tomaron los nombres de las preparaciones y los alimentos consumidos un día antes, desde que la persona se despertó hasta que terminó su día, como una biografía de los alimentos y bebidas preparados, comprados y consumidos.

A pesar de que el recordatorio de 24 horas no nos describe la dieta habitual del individuo, es altamente probable que ésta sea la alimentación cotidiana de la familia. Por este motivo el recordatorio se realizó en un día normal, es decir, no se trató de un día festivo o fin de semana, que es cuando suelen modificarse los alimentos y prácticas usuales. Por otro lado también se consultó a las madres de familia acerca del uso del micronutriente CHIS PAZ. Debido a esto solamente se trabajó con las personas encargadas de preparar los alimentos, para este caso generalmente fueron madres con niños y niñas menores de cinco años. 


\section{Resultados}

Los resultados de los recordatorios en la comunidad de San Clemente evidenciaron, el consumo de fréjol y arroz en la dieta. Las tres mujeres, los niños y las niñas (a través de la información provista por sus madres) muestran los siguientes resultados:

\begin{tabular}{|c|c|c|c|}
\hline \multicolumn{2}{|r|}{ MADRE } & \multicolumn{2}{|r|}{ NIÑO/A } \\
\hline \multicolumn{4}{|c|}{ DESAYUNO } \\
\hline 1 & $\begin{array}{l}\text { a) leche, azúcar } \\
\text { b) pan } \\
\text { c) huevo, sal } \\
\text { d) arroz, sal, aceite }\end{array}$ & 1 & $\begin{array}{l}\text { a) medio huevo } \\
\text { b) jugo de naranja, azúcar } \\
\text { c) leche materna }\end{array}$ \\
\hline 2 & $\begin{array}{l}\text { a) avena } \\
\text { b) naranjilla, agua, azúcar } \\
\text { c) pan } \\
\text { d) huevo, sal }\end{array}$ & 2 & $\begin{array}{l}\text { a) avena } \\
\text { b) naranjilla, azúcar } \\
\text { c) pan } \\
\text { d) huevo, sal }\end{array}$ \\
\hline 3 & $\begin{array}{l}\text { a) café, azúcar } \\
\text { b) pan, mermelada, queso. } \\
\text { c) huevo, sal. } \\
\text { d) jugo de tomate de árbol, azúcar }\end{array}$ & 3 & $\begin{array}{l}\text { a) café, azúcar } \\
\text { b) pan, mermelada, queso } \\
\text { c) huevo, sal } \\
\text { d) medio jugo de tomate de árbol, azúcar }\end{array}$ \\
\hline \multicolumn{4}{|c|}{ MEDIA MÃ̃ ANA } \\
\hline 1 & a) ningún alimento & 1 & a) ningún alimento \\
\hline 2 & $\begin{array}{l}\text { a) guineos } \\
\text { b) manzana }\end{array}$ & 2 & $\begin{array}{l}\text { a) guineos } \\
\text { b) manzana }\end{array}$ \\
\hline 3 & a) ningún alimento & 3 & a) ningún alimento \\
\hline \multicolumn{4}{|c|}{ ALMUERZO } \\
\hline 1 & $\begin{array}{l}\text { a) fréjol, cebolla larga, sal, aceite, ajo } \\
\text { b) arroz, aceite, sal } \\
\text { c) avena, sal, papa, cebolla larga, ajo, achiote } \\
\text { d) limón, azúcar }\end{array}$ & 1 & $\begin{array}{l}\text { a) avena, sal, papa, cebolla larga, ajo, achiote } \\
\text { b) leche materna (cada dos horas) }\end{array}$ \\
\hline 2 & $\begin{array}{l}\text { a) fréjol, tomate, sal, cebolla larga, cilantro } \\
\text { b) arroz, aceite, sal, plátano } \\
\text { c) limón, panela }\end{array}$ & 2 & $\begin{array}{l}\text { a) fréjol, tomate, sal, cebolla larga, cilantro } \\
\text { b) arroz, aceite, sal } \\
\text { c) plátano } \\
\text { d) jugo de limón, panela }\end{array}$ \\
\hline 3 & $\begin{array}{l}\text { a) lentejas, sal, papa, zanahoria, cebolla, carne } \\
\text { de cerdo, fideos, cilantro, comino, pimienta, } \\
\text { cubito }\end{array}$ & 3 & $\begin{array}{l}\text { a) (medio plato) lentejas, sal, papa, zanahoria, } \\
\text { cebolla, carne de cerdo, fideos, cilantro, } \\
\text { comino, pimienta, cubito }\end{array}$ \\
\hline \multicolumn{4}{|c|}{ MEDIA TARDE } \\
\hline 1 & a) naranja & 1 & a) leche materna \\
\hline 2 & a) sandía & 2 & $\begin{array}{l}\text { a) sandia. } \\
\text { b) canguil, sal } \\
\text { c) café, azúcar }\end{array}$ \\
\hline 3 & a) ningún alimento & 3 & a) manzana \\
\hline
\end{tabular}




\begin{tabular}{|c|l|c|l|}
\hline \multicolumn{3}{|c|}{ CENA } \\
\hline 1 & $\begin{array}{l}\text { a) fréjol, cebolla larga, sal, aceite, ajo } \\
\text { b) arroz, aceite, sal } \\
\text { c) avena, sal, papa, cebolla larga, ajo, achiote } \\
\text { d) limón, azúcar }\end{array}$ & 1 & $\begin{array}{l}\text { a) avena, sal, papa, cebolla larga, ajo, achiote } \\
\text { b) leche materna (cada dos horas). } \\
\text { c) CHIS PAZ }\end{array}$ \\
\hline 2 & $\begin{array}{l}\text { a) arroz, sal, choclo, aceite, sal } \\
\text { b) jugo de tomate de árbol, azúcar }\end{array}$ & 2 & $\begin{array}{l}\text { a) arroz, sal, choclo, aceite, sal } \\
\text { b) jugo de tomate de árbol, azúcar }\end{array}$ \\
\hline 3 & $\begin{array}{l}\text { a) arroz, aceite, sal } \\
\text { b) papas fritas, aceite, sal } \\
\text { c) cebolla, tomate, limón, cilantro, sal } \\
\text { d) huevo frito, aceite, sal } \\
\text { e) jugo de naranjilla }\end{array}$ & 3 & $\begin{array}{l}\text { a) (medio plato) arroz, aceite, sal } \\
\text { b) papas fritas, aceite, sal } \\
\text { c) cebolla, tomate, limón, cilantro, sal } \\
\text { d) huevo frito, aceite, sal }\end{array}$ \\
\hline
\end{tabular}

Elaboración propia

Por su parte la Tabla 2, representa los grupos de alimentos consumidos tanto en las madres como en los niños y niñas menores de cinco años.

\begin{tabular}{|c|c|c|c|c|c|}
\hline \multicolumn{3}{|r|}{ MADRE } & \multicolumn{3}{|r|}{ NIÑO/A } \\
\hline 1 & $\begin{array}{l}\text { a) } \\
\text { b) } \\
\text { c) } \\
\text { d) } \\
\text { e) } \\
\text { f) } \\
\text { g) } \\
\text { h) } \\
\text { i) } \\
\text { j) }\end{array}$ & $\begin{array}{l}\text { Granos y tubérculos (9) } \\
\text { Carnes (0) } \\
\text { Azúcar (4) } \\
\text { Frutas y vegetales (16) } \\
\text { Lácteos (1), } \\
\text { Aceites y grasas (6) } \\
\text { Leguminosas (3) } \\
\text { Huevos (1) } \\
\text { Sal (9) } \\
\text { Otros (2) }\end{array}$ & 1 & $\begin{array}{l}\text { a) } \\
\text { b) } \\
\text { c) } \\
\text { d) } \\
\text { e) } \\
\text { f) } \\
\text { g) } \\
\text { h) } \\
\text { i) } \\
\text { j) } \\
\text { k) }\end{array}$ & $\begin{array}{l}\text { Granos y tubérculos (5) } \\
\text { Carnes (0) } \\
\text { Azúcar (2) } \\
\text { Frutas y vegetales (10) } \\
\text { Lácteos - leche materna (4) } \\
\text { Aceites y grasas (1) } \\
\text { Leguminosas (1) } \\
\text { Huevos (1) } \\
\text { Sal (4) } \\
\text { Otros (2) } \\
\text { CHIS PAZ (1) }\end{array}$ \\
\hline 2 & $\begin{array}{l}\text { a) } \\
\text { b) } \\
\text { c) } \\
\text { d) } \\
\text { e) } \\
\text { f) } \\
\text { g) } \\
\text { h) } \\
\text { i) } \\
\text { j) }\end{array}$ & $\begin{array}{l}\text { Granos y tubérculos (4) } \\
\text { Carnes (0) } \\
\text { Azúcar (2) } \\
\text { Frutas y vegetales (5) } \\
\text { Lácteos (0) } \\
\text { Aceites y grasas (1) } \\
\text { Leguminosas (0) } \\
\text { Huevo (1) } \\
\text { Sal (3) } \\
\text { Otros (0) }\end{array}$ & 2 & $\begin{array}{l}\text { a) } \\
\text { b) } \\
\text { c) } \\
\text { d) } \\
\text { e) } \\
\text { f) } \\
\text { g) } \\
\text { h) } \\
\text { i) } \\
\text { j) }\end{array}$ & $\begin{array}{l}\text { Granos y tubérculos (5) } \\
\text { Carnes (0) } \\
\text { Azúcar (3) } \\
\text { Frutas y vegetales (5) } \\
\text { Lácteos (0) } \\
\text { Aceites y grasas (1) } \\
\text { Leguminosas (0) } \\
\text { Huevo (1) } \\
\text { Sal (4) } \\
\text { Otros (1) }\end{array}$ \\
\hline 3 & $\begin{array}{l}\text { a) } \\
\text { b) } \\
\text { c) } \\
\text { d) } \\
\text { e) } \\
\text { f) } \\
\text { g) } \\
\text { h) } \\
\text { i) } \\
\text { j) }\end{array}$ & $\begin{array}{l}\text { Granos y tubérculos (5) } \\
\text { Carnes (1) } \\
\text { Azúcar (3) } \\
\text { Frutas y vegetales (8) } \\
\text { Lácteos.(1) } \\
\text { Aceites y grasas (3) } \\
\text { Leguminosas (1) } \\
\text { Huevo (2) } \\
\text { Sal (6) } \\
\text { Otros (4) }\end{array}$ & 3 & $\begin{array}{l}\text { a) } \\
\text { b) } \\
\text { c) } \\
\text { d) } \\
\text { e) } \\
\text { f) } \\
\text { g) } \\
\text { h) } \\
\text { i) } \\
\text { j) }\end{array}$ & $\begin{array}{l}\text { Granos y tubérculos (5) } \\
\text { Carnes (1) } \\
\text { Azúcar (3) } \\
\text { Frutas y vegetales (8) } \\
\text { Lácteos.(1) } \\
\text { Aceites y grasas (3) } \\
\text { Leguminosas (1) } \\
\text { Huevo (2), } \\
\text { Sal (6) } \\
\text { Otros (4) }\end{array}$ \\
\hline
\end{tabular}

Elaboración propia / (n) Representa la cantidad de alimentos de ese grupo alimentario. 
A pesar de que en los grupos de alimentos se observa diversidad en la dieta, según señala Calero (2011: 34) en sus escritos, estos alimentos podrían ser adquiridos a través de "compras [,] producción propia, intercambio de alimentos, regalos o donaciones". En hogares de la Sierra y Costa los alimentos provienen de la adquisición a través de mecanismos monetarios, sin embargo en regiones como la Amazonía los alimentos vienen principalmente del autoconsumo.

Así mismo Calero califica a los hogares con inseguridad alimentaria cuando estos tienen un mayor número de niños menores de cinco años (Ibíd.:38). El mismo autor enfatiza que los hogares con menor acceso y disponibilidad de alimentos, son aquellos que destinan mayor porcentaje de sus ingresos al gasto para la alimentación, a diferencia de los hogares que son seguros en términos alimentarios (Ibíd.:39), tal y como lo vimos a través de la ENIGHUR.

A través de los resultados, los cuales muestran dietas diversas, podemos decir que los hogares en cuestión no sufren de inseguridad alimentaria. Sin embargo si utilizamos la tipología alimentaria citada por Messer (2006), vemos que estos hogares poseen dietas básicas o casi vegetarianas. En términos de justicia social este tipo de dieta alimentaria a un mayor número de personas. Sin embargo sigue siendo básica. San Clemente se trata de una dieta básica, en la que no existe mayor cantidad de alimentos de origen animal. Como se observa en la Tabla 2., cuando se hace la clasificación por grupos de alimentos, la proteína proviene principalmente de alimentos de origen vegetal, por lo que la autora asegura que este tipo de dieta es "casi vegetariana" (Messer, 2006: 32).

\section{El programa CHIS PAZ}

A partir de los 90' en adelante se creyó que las sociedades debían tener un pensamiento global y éste debía ser aplicado en lo local y no lo contrario (Ibíd.: 29). Esto se vincula con las formas en las que se ha hecho intervención en términos de nutrición y alimentación en poblaciones vulnerables. No obstante no se conoce en su totalidad la realidad local, ni las formas de adopción de estas intervenciones, mismas que en el caso de CHIS PAZ se da a través de suplementos y/o micronutrientes.

Para enfrentar la desnutrición por anemia en niños y niñas menores de cinco años, el Ministerio de Inclusión Económica y Social (MIES) distribuye en los centros de salud el micronutriente CHIS PAZ, y estos a su vez proveen a las madres de familia. Este micronutriente presenta alto contenido de hierro, zinc, vitaminas A y $\mathrm{C}$ y ácido fólico. Los niños y niñas deben consumir un sobre diario durante 60 días. Para su aplicación se debe tener especial cuidado en la forma de preparación, en un alimento tibio, no caliente, y en alimentos espesos; el consumo debe ser inmediato debido que al pasar media hora, el sabor del alimento se torna metálico y oscuro. Este micronutriente fue desarrollado para reemplazar el uso de las gotas de hierro (Oller de Daroca, 2004; Zlotkin, 2004 en MSP, 2011: 26). CHIS PAZ tiene el objetivo de disminuir los niveles de anemia y es proveído a las madres de familia y promotoras de salud con previa capacitación, con el objetivo de que haya un buen manejo del mismo.

A través de las conversaciones con las madres de familia se pudo reconocer su posición frente al suplemento vitamínico CHIS PAZ. AR1SC, una de las madres aseguro lo siguiente:

"Las chis paz son ácidas, no le doy todo el sobre, le doy sólo la mitad, porque si no el niño no se come todo porque la comida se pone fea" (AR1SC, 2013, Entrevista).

Por otro lado, la tercera madre con quien se mantuvo conversaciones, manifestó lo siguiente, respecto a las CHIS PAZ:

"La leche materna es importante para que no se enferme la wawa...las chispaz son para niños que tienen anemia, a los niños chiquitos si les dan chispaz, pero a mi hija no le dan chispaz... yo he probado porque a mi sobrino le dan, y como yo le doy de lactar a él [su sobrino]....pero es ácido, eso no debería ser para niños....mi hija no toma, pero les dan dos sobres al día" (NA3SC, 2013, Entrevista). 
Las intervenciones más conocidas para mejorar la nutrición se dan a través de asistencia alimentaria, con la distribución de alimentos fortificados, suplementos, micronutrientes, etc. (Calero, 2011: 67). Cabe aclarar que según el mismo autor, los suplementos y alimentos fortificados son la manera más barata de paliar la malnutrición ${ }^{2}$.

Los programas de distribución de micronutrientes como las CHIS PAZ se ofertan luego de una capacitación para la preparación de los mismos a través de charlas en los centros de salud. A pesar de ello, en el caso de las madres de familia con quienes realizamos los recordatorios, ellas no se apropian de esta preparación, por lo tanto, según los testimonios, al niño pareciera no gustarle los micronutrientes administrados en el hogar. Por otro lado hay madres que poseen un conocimiento empírico, como por ejemplo la segunda madre, quien manifestó lo siguiente:

"Si le doy Chis Paz a mi hijo, yo no he probado, pero parece que le gusta". (LI2SC, 2013, Entrevista).

Podemos argumentar, sobre la base de la información que disponemos, que la prevalencia en el uso de micronutrientes destinados a reducir la anemia se debe a que la dieta se basa principalmente en alimentos que poseen hierro de origen vegetal. Al respecto algunos autores aseguran:

[...] A pesar del alto contenido de Fe-No Hem de los alimentos, su biodisponibilidad varía desde menos del $1 \%$ hasta un $20 \%$, esto se debe a que otros nutrientes de la dieta pueden aumentar o disminuir la eficiencia [...] (Gaitán y otros, 2006).

[...] El hierro hemínico se encuentra regularmente en una dieta estándar entre 10 y $20 \%$, mientras que el no hemínico se encuentra en mayores proporciones (80 a 90\%). A pesar de esto, el grupo hemo alcanza más de 50\% de absorción mientras que el hierro no hemínico sólo de 1 a $10 \%$ [...] (Tostado - Madrid y otros, 2015: 194).

La biodisponibilidad del hierro no hemínico es baja en alimentos de origen vegetal, en leche y en huevos (Gaitán y otros, 2006). Esta biodisponilidad reducida ocasiona que los niños que no han sido alimentados con alimentos de alta biodisponibilidad de hierro presenten deficiencias productivas en la edad adulta. Los problemas relacionados con la baja disponibilidad de hierro hemínico son más frecuentes en países en vías de desarrollo, por el acceso reducido a los alimentos de origen animal, como las carnes. Esto se debe a que las dietas de países en vías de desarrollo existe más prominencia de alimentos como legumbres y cereales (Gaitán y otros, 2006: 2 - 40), con dietas generalmente monótonas. Cabe aclarar que no se está desacreditando a las dietas vegetarianas. Sin embargo estas últimas requieren de planeación a fin de evitar la carencia de micronutrientes como el hierro y otros de baja biodisponibilidad; Así como prevenir o contrarrestar la presencia de inhibidores de estos micronutrientes.

\section{Conclusiones}

Los sistemas agroalimentarios a pesar de modificarse a favor de la agricultura comercial, podrían afectar de igual manera la nutrición de las comunidades rurales. Por medio de las respuestas de las madres e hijos se pudo evidenciar que tienen una dieta diversificada.

La dieta diversificada según la tipología alimentaria es catalogada como una dieta básica, es decir, una dieta con ausencia de alimentos de origen animal. Se puede evidenciar que el hierro de origen vegetal es el de mayor consumo, a través de vegetales verdes y leguminosas como el fréjol.

Por otro lado, la intervención para prevenir la desnutrición por deficiencia de hierro en niños y niñas menos de cinco años es, sin duda, bien intencionada. Pese a ello las madres no adoptan

2 Se refiere a las diferentes formas de nutrición deficiente originada por una alimentación inadecuada, enfermedades infecciosas y otras (Banco Mundial, 2006) citado en (Calero, 2011: 68) 
estas medidas de la manera en que se recomienda. Se comprobó que hay cierto rechazo por parte de los niños y niñas; y una percepción de este mismo patrón en las madres, principalmente por el sabor. Pese a que el programa CHIS PAZ goza de una distribución adecuada en las áreas rurales del Ecuador, a nivel de centros de salud y promotores, el programa disminuye su impacto al momento en que llega a los hogares, y específicamente para nuestro caso de estudio, cuando llega a las cuidadoras de los niños y niñas menores de cinco años. Por lo tanto la inversión realizada para la investigación y desarrollo de micronutrientes de alta biodisponibilidad de hierro hemínico, resulta en pérdidas al momento de la aplicación en los hogares. Como se vio, dos de los tres casos expuestos, muestran un rechazo debido a la "práctica inadecuada" frente a este tipo de productos. Lo anterior debido al desconocimiento de la preparación; o al no reconocimiento de estos micronutrientes como comida, como es el caso de la primera madre.

La ausencia de alimentos de origen animal, y por ende la baja biodisponibilidad de hierro hemínico podría tratarse de un hecho común en la comunidad. La presencia de carne de cerdo en la tercera madre, podría no ser un hecho común en la dieta de la población, pero es posible que sea una estrategia ante la baja disponibilidad de hierro no hemínico en el resto de los alimentos en este caso en particular.

Asimismo podemos observar que existe una alta dependencia de productos como la sal, aceite y azúcar. Se podría decir que las familias que adquieren sus alimentos a través de transacciones comerciales, son aquellas más dependientes de alimentos del mercado, y presentan menor capacidad de producción de alimentos para el autoconsumo, factor que debilita la soberanía alimentaria de las áreas rurales.

Finalmente, es importante reiterar que las poblaciones necesitan ser entendidas a nivel de la localidad, a nivel de comunidades, hogares e individuos, porque es aquí donde se pueden encontrar importantes estrategias para enfrentar los problemas relacionados con la malnutrición. Por otro lado, es importante identificar de qué manera son adoptadas las intervenciones globales en la localidad, como es el caso del programa CHIS PAZ.

\section{Bibliografía}

AGRECO. 2010, Informe Proyecto Población Afro y Mestiza del Norte del Ecuador Consolidando Procesos de Seguridad Alimentaria (PAMECSA).

Calero, C. J. 2011, Seguridad alimentaria en Ecuador desde un enfoque de acceso a alimentos, FLACSO - Abya Yala, Quito.

Food and Agriculture Organization (FAO). 1969, Plan indicatif mondial provioire pour le Développment de l' Agriculture, FAO, Roma.

Gaitán, D., Olivares, M., Arredondo, M., \& Pizarro, A. 2006, "Biodisponibilidad de hierro en humanos", en: Revista Chilena de Nutrición, pp.142-148.

Garine, I. d. 2002, "Los aspectos socioculturales de la nutrición", en: J. Contreras, Alimentación y cultura. Necesidades, gustos y costumbres, Ediciones UB, Barcelona, pp. 129-169.

Garine, I. d. 1962, Usages alimentaires dans la Région de Khomboile (Sénegal). Cahiers d’Études Africanes.

Instituto Nacional de Estadísticas y Censos (INEC). 2008, La desnutrición en la población indígena y agroeconuatoriana menor de cinco años. Análisis Sociodemográfico, INEC, Quito.

Messer, E. 2006, Globalización y dieta: significados, cultura y consecuencias en la nutrición, en: Antropología y Nutrición, pp. 27 - 74.

Ministerio de Salud Pública (MSP). 2011, Normas, protocolos y consejería para la suplementación con micronutrientes, MSP, Quito.

Oller de Daroca, M. 2004, Producción y distribución de fortificantes caseros para alimentos infantiles: Chispitas nutricionales para los más pequeños, Organización Panamericana y Mundial de la Salud (OPS), La Paz.

Poulain, J. P. 2008, Manger Aujourf'hui: attitudes, normes et pratiques. Toulouse Privat.

Soto, M. 2011, Diagnóstico inicial Nutrición y Agua. Parroquia Ambuqui. Comunidad San Clemente, pp. 1-11.

Tostado-Madrid, T., Benítez-Ruíz, I., Pinzón-Navarro, A., Bautista-Silva, M., \& Ramírez-Mayans, J. 2015, "Actualidades de las características del hierro y su uso en pediatría", en: Acta Pediátria de México, pp. 189-200. 